\title{
A Double S-Shaped Bifurcation Curve for a Reaction-Diffusion Model with Nonlinear Boundary Conditions
}

\author{
Jerome Goddard II, ${ }^{1}$ Eun Kyoung Lee, ${ }^{2}$ and R. Shivaji ${ }^{1}$ \\ ${ }^{1}$ Department of Mathematics and Statistics, Center for Computational Sciences, \\ Mississippi State University, Mississippi State, MS 39762, USA \\ ${ }^{2}$ Department of Mathematics, Pusan National University, Busan 609-735, Republic of Korea
}

Correspondence should be addressed to R. Shivaji, shivaji@ra.msstate.edu

Received 13 November 2009; Accepted 23 May 2010

Academic Editor: Martin D. Schechter

Copyright (C) 2010 Jerome Goddard II et al. This is an open access article distributed under the Creative Commons Attribution License, which permits unrestricted use, distribution, and reproduction in any medium, provided the original work is properly cited.

\begin{abstract}
We study the positive solutions to boundary value problems of the form $-\Delta u=\lambda f(u)$; $\Omega$, $\alpha(x, u)(\partial u / \partial \eta)+[1-\alpha(x, u)] u=0 ; \partial \Omega$, where $\Omega$ is a bounded domain in $\mathbb{R}^{n}$ with $n \geq 1, \Delta$ is the Laplace operator, $\lambda$ is a positive parameter, $f:[0, \infty) \rightarrow(0, \infty)$ is a continuous function which is sublinear at $\infty, \partial u / \partial \eta$ is the outward normal derivative, and $\alpha(x, u): \Omega \times \mathbb{R} \rightarrow[0,1]$ is a smooth function nondecreasing in $u$. In particular, we discuss the existence of at least two positive radial solutions for $\lambda \gg 1$ when $\Omega$ is an annulus in $\mathbb{R}^{n}$. Further, we discuss the existence of a double S-shaped bifurcation curve when $n=1, \Omega=(0,1)$, and $f(s)=e^{\beta s /(\beta+s)}$ with $\beta \gg 1$.
\end{abstract}

\section{Introduction}

In this paper, we consider the reaction-diffusion model with nonlinear boundary condition given by

$$
\begin{gathered}
u_{t}=d \Delta u+\lambda f(u) ; \quad \Omega, \\
d \alpha(x, u) \frac{\partial u}{\partial \eta}+[1-\alpha(x, u)] u=0 ; \quad \partial \Omega,
\end{gathered}
$$

where $\Omega$ is a bounded domain in $\mathbb{R}^{n}$ with $n \geq 1, \Delta$ is the Laplace operator, $\lambda$ is a positive parameter, $d$ is the diffusion coefficient, $\partial u / \partial \eta$ is the outward normal derivative, $f:[0, \infty) \rightarrow$ $(0, \infty)$ is a smooth function, and $\alpha(x, u): \Omega \times \mathbb{R} \rightarrow[0,1]$ is a smooth function nondecreasing 
in $u$. The boundary condition (1.2) arises naturally in applications and has been studied by the authors of [1-4], among others, in particular in the context of population dynamics. Here

$$
\alpha(x, u)=\frac{u}{u-d(\partial u / \partial \eta)}
$$

represents the fraction of the substance that $u(x)$ represents that remains at the boundary when reached. In particular, we will be interested in the study of positive steady state solutions of (1.1)-(1.2) when $d=1$, namely,

$$
\begin{gathered}
-\Delta u=\lambda f(u) ; \quad \Omega \\
\alpha(x, u) \frac{\partial u}{\partial \eta}+[1-\alpha(x, u)] u=0 ; \quad \partial \Omega
\end{gathered}
$$

with $f(u)$ satisfying

$$
\begin{aligned}
& \left(H_{1}\right) \lim _{u \rightarrow \infty}(f(u) / u)=0, \\
& \left(H_{2}\right) M:=\inf _{u \in[0, \infty)}\{f(u)\}>0 .
\end{aligned}
$$

The motivating example for this study comes from combustion theory (see [5-15]) where $f(u)$ takes the form:

$$
f(u)=e^{\beta u /(\beta+u)}
$$

with positive parameter $\beta$. When $\alpha(x, u) \equiv 0$ (Dirichlet boundary condition case) there is already a very rich history in the literature about positive solutions of (1.4)-(1.5). In particular, when $f(u)=e^{\beta u /(\beta+u)}$ and $\beta \gg 1$ the bifurcation diagram of positive solutions is known to be S-shaped (see $[16,17])$. The main purpose of this paper is to extend this study to the nonlinear boundary condition (1.5), namely, when

$$
\alpha(x, u)=\frac{u}{u+1}
$$

on part of the boundary.

Firstly, we discuss the case when $n>1, \Omega=\left\{x \in \mathbb{R}^{n}\left|R_{1} \leq\right| x \mid \leq R_{2}\right\}$ is an annulus in $\mathbb{R}^{n}, n \geq 1$, and

$$
\alpha(x, u)= \begin{cases}0, & |x|=R_{1} \\ \frac{u}{u+1}, & |x|=R_{2} .\end{cases}
$$

In Section 2, we show that if $\left(H_{1}\right)$ and $\left(H_{2}\right)$ both hold, then there exists $\lambda^{*}>0$ such that

(i) for $0<\lambda \leq \lambda^{*},(1.4)$-(1.5) has a positive radial solution;

(ii) for $\lambda>\lambda^{*}$, (1.4)-(1.5) has at least two positive radial solutions. 
Secondly, we present results for the case when $n=1, \Omega=(0,1), f(u)=e^{\beta u /(\beta+u)} ; \beta>0$, and

$$
\alpha(x, u)= \begin{cases}0, & x=0 \\ \frac{u}{u+1}, & x=1 .\end{cases}
$$

Thus, we study the nonlinear boundary value problem

$$
\begin{gathered}
-u^{\prime \prime}=\lambda e^{\beta u /(\beta+u)}, \quad x \in(0,1), \\
u(0)=0 \\
\frac{u(1)}{u(1)+1}\left[-u^{\prime}(1)\right]+\left[1-\frac{u(1)}{u(1)+1}\right] u(1)=0 .
\end{gathered}
$$

Clearly, studying (1.10) is equivalent to analyzing the two boundary value problems

$$
\begin{gathered}
-u^{\prime \prime}=\lambda e^{\beta u /(\beta+u)}, \quad x \in(0,1), \\
u(0)=0, \\
u(1)=0, \\
-u^{\prime \prime}=\lambda e^{\beta u /(\beta+u)}, \quad x \in(0,1), \\
u(0)=0, \\
u^{\prime}(1)=-1 .
\end{gathered}
$$

In particular, the positive solutions of (1.11) and (1.12) are the positive solutions of (1.10). In Section 3 we present Quadrature methods used to completely determine the bifurcation diagrams of (1.11) and (1.12), respectively. We show that for $\beta$ large enough, (1.10) has a double S-shaped bifurcation curve with exactly 6 positive solutions for a certain range of $\lambda$ (see Figure 1).

\section{Existence and Multiplicity Results when $\Omega$ is an Annulus in $\mathbb{R}^{n}$ and $n \geq 1$}

Here we consider the existence of positive radial solutions for

$$
\begin{gathered}
-\Delta u=\lambda f(u), \quad \Omega \\
\alpha(x, u) \frac{\partial u}{\partial \eta}+[1-\alpha(x, u)] u=0, \quad \partial \Omega
\end{gathered}
$$




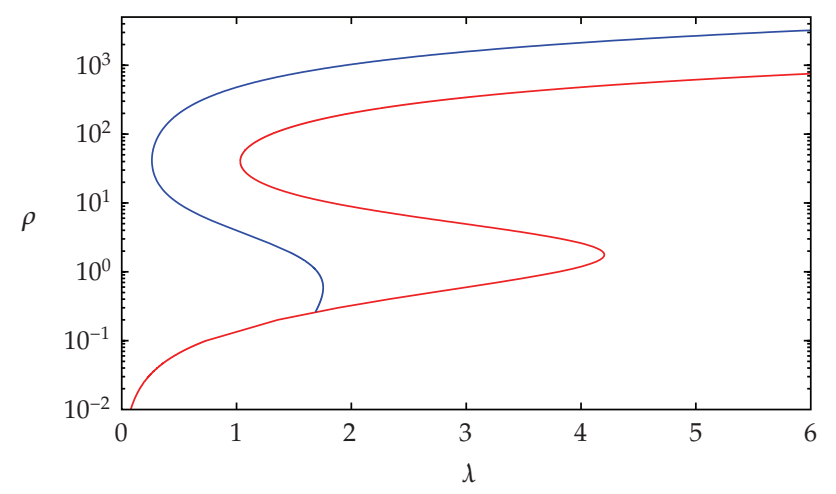

Figure 1: Double S-shaped bifurcation curve.

when $f:[0, \infty) \rightarrow(0, \infty)$ is a continuous function, $\Omega=\left\{x \in \mathbb{R}^{n}\left|R_{1}<\right| x \mid<R_{2}, 0<R_{1}<R_{2}\right\}$, and

$$
\alpha(u)= \begin{cases}0 & \text { if }|x|=R_{1} \\ \frac{u}{u+1} & \text { if }|x|=R_{2} .\end{cases}
$$

Then the boundary condition of (2.1) is

$$
\begin{array}{r}
u=0 \quad \text { if }|x|=R_{1}, \\
u\left[\frac{\partial u}{\partial \eta}+1\right]=0 \quad \text { if }|x|=R_{2} .
\end{array}
$$

Thus to obtain positive solutions for (2.1), we study

$$
\begin{aligned}
& -\Delta u=\lambda f(u) \text { in } \Omega, \\
& u=0 \quad \text { on } \partial \Omega, \\
& -\Delta u=\lambda f(u) \text { in } \Omega, \\
& u=0 \quad \text { if }|x|=R_{1}, \\
& \frac{\partial u}{\partial \eta}=-1 \quad \text { if }|x|=R_{2} .
\end{aligned}
$$

The existence of positive solutions of (2.4) follows from $[16,18]$ in the following theorem.

Theorem 2.1 (see $[16,18]$ ). Assume $\left(H_{1}\right)$. Then (2.4) has a positive radial solution for all $\lambda>0$. 
Now we consider radial solutions to the problem (2.5). Let

$$
m=-\int_{R_{1}}^{R_{2}} \frac{1}{\tau^{n-1}} d \tau
$$

By applying consecutive changes of variables, $r=|x|, s=-\int_{r}^{R_{2}}\left(1 / \tau^{n-1}\right) d \tau, t=(m-s) / m$, and $z(t)=u(r)=u(|x|),(2.5)$ is equivalently transformed into the problem

$$
\begin{gathered}
-z^{\prime \prime}(t)=\lambda h(t) f(z(t)) \quad 0<t<1, \\
z(0)=0, \quad z^{\prime}(1)=-b,
\end{gathered}
$$

where

$$
\begin{gathered}
b=-m R_{2}^{n-1}>0, \\
h(t)=m^{2}[r(m(1-t))]^{2(n-1)} .
\end{gathered}
$$

Note that $h:[0,1] \rightarrow[0, \infty)$ is continuous function. For details about this transformation, see [19]. We prove the existence of a positive solution of (2.7) by using the fixed point index in a cone. This fixed point index is equivalent to the Leray-Schauder degree which means that if $K$ is a cone in a Banach space $E, \mathcal{O}$ is bounded and open in $E, 0 \in \mathcal{O}$, and $T: K \cap \overline{\mathcal{O}} \rightarrow K$ is completely continuous then

$$
i(T, K \cap \mathcal{O}, K)=\operatorname{deg}\left(\mathrm{id}-T \circ r, r^{-1}(K \cap \mathcal{O}), 0\right),
$$

where $r: E \rightarrow K$ is an arbitrary retraction (see [20]).

Lemma A (see [21]). Let E be a Banach space, $K$ a cone in $E$ and $\mathcal{O}$ bounded open in E. Let $0 \in \mathcal{O}$, and let $T: K \cap \overline{\mathcal{O}} \rightarrow K$ be completely continuous. Suppose that $T x \neq v x$, for all $x \in K \cap \partial \mathcal{O}$ and all $v \geq 1$. Then

$$
i(T, K \cap \mathcal{O}, K)=1 .
$$

Define $T_{\lambda}: C[0,1] \rightarrow C[0,1]$ by

$$
T_{\lambda} z(t)=-b t+\lambda \int_{0}^{1} G(t, s) h(s) f(z(s)) d s
$$

where

$$
G(t, s)= \begin{cases}t, & 0 \leq t \leq s \leq 1 \\ s, & 0 \leq s \leq t \leq 1\end{cases}
$$


Then $T_{\lambda}: C[0,1] \rightarrow C[0,1]$ is completely continuous and $u$ is a solution of $(2.7)$ if and only if $u$ is a fixed point of $T_{\lambda}$, that is, $T_{\lambda} u=u$.

Theorem 2.2. If $\left(H_{1}\right)$ and $\left(H_{2}\right)$ both hold then (2.7) has a positive solution for all $\lambda>$ $b / M \int_{0}^{1} s h(s) d s$, where $b$ and $h(t)$ are defined as in (2.8).

Proof. Define $K:=\{z \in C[0,1] \mid z(t) \geq 0, t \in[0,1]$ and $z$ is concave $\}$, then $K$ is a cone in $C[0,1]$. Further if $\lambda>b / M \int_{0}^{1} \operatorname{sh}(s) d s$, then $T_{\lambda}(K) \subset K$. In fact, if $z \in K$, then it is easy to show that $T_{\lambda} z(0)=0$ and $T_{\lambda} z^{\prime \prime}(t) \leq 0$ for $t \in[0,1]$. Also if $\lambda>b / M \int_{0}^{1} \operatorname{sh}(s) d s$, we have

$$
\begin{aligned}
T_{\lambda} z(1) & =-b+\lambda \int_{0}^{1} \operatorname{sh}(s) f(z(s)) d s \\
& \geq-b+\lambda M \int_{0}^{1} \operatorname{sh}(s) d s>0 .
\end{aligned}
$$

Thus $T_{\lambda}(z) \in K$. have

Define $\tilde{f}(z):=\max _{t \in[0, z]} f(t)$. Then $f(z) \leq \tilde{f}(z), \tilde{f}$ is nondecreasing, and from $\left(H_{1}\right)$, we

$$
\lim _{z \rightarrow \infty} \frac{\tilde{f}(z)}{z}=0
$$

Fix $\rho_{\lambda} \in\left(0,1 / \lambda \int_{0}^{1} s h(s) d s\right)$. From (2.14), there is $m_{\curlywedge}>0$ such that

$$
\tilde{f}(z) \leq \rho_{\curlywedge} z \quad \forall z \geq m_{\curlywedge}
$$

Let $\mathcal{O}_{\lambda}:=\left\{z \in C[0,1] \mid\|z\|_{\infty}<m_{\lambda}\right\}$. Then $\mathcal{O}_{\lambda}$ is bounded and open in $C[0,1], 0 \in \mathcal{O}_{\lambda}$, and $T_{\lambda}: K \cap \overline{\mathcal{O}_{\lambda}} \rightarrow K$ is completely continuous. If $z \in K \cap \partial \mathcal{O}_{\lambda}$, then from monotonicity of $\tilde{f}$ and (2.15) we have

$$
\begin{aligned}
T_{\lambda} z(t) & \leq \lambda \int_{0}^{1} \operatorname{sh}(s) \tilde{f}(z(s)) d s \\
& \leq \lambda \tilde{f}\left(m_{\curlywedge}\right) \int_{0}^{1} \operatorname{sh}(s) d s \\
& \leq \lambda \rho_{\lambda} m_{\curlywedge} \int_{0}^{1} \operatorname{sh}(s) d s \\
& <m_{\curlywedge}=\|z\|_{\infty} .
\end{aligned}
$$

Thus $T_{\lambda} z \neq v z$ for all $v \geq 1$. Now applying Lemma A, we have

$$
i\left(T_{\lambda}, K \cap \mathcal{O}_{\lambda}, K\right)=1,
$$

which means that $T_{\lambda}$ has a fixed point in $K \cap \mathcal{O}_{\lambda}$. Thus Theorem 2.2 is proven. 
Further, if we additionally assume that

$\left(H_{3}\right) N:=\sup _{u \in[0, \infty)}\{f(u)\}<\infty$, then we can show nonexistence for $\lambda \ll 1$.

Theorem 2.3. If $\left(\mathrm{H}_{3}\right)$ holds then (2.7) has no positive solution for all $\lambda<b / N \int_{0}^{1} \operatorname{sh}(s) d s$, where $b$ and $h(t)$ are defined as in (2.8).

Proof. Suppose that $u_{\curlywedge}$ is a positive solution of (2.7). Thus,

$$
\begin{aligned}
u_{\lambda}(t)=T_{\lambda} u_{\lambda}(t) & =-b t+\lambda \int_{0}^{1} G(t, s) h(s) f\left(u_{\lambda}(s)\right) d s \\
& \leq-b t+\lambda N \int_{0}^{1} G(t, s) h(s) d s .
\end{aligned}
$$

Letting $t=1$ gives

$$
u_{\lambda}(1) \leq-b+\lambda N \int_{0}^{1} \operatorname{sh}(s) d s
$$

Since $u_{\lambda}(t)$ is positive, we have

$$
-b+\lambda N \int_{0}^{1} \operatorname{sh}(s) d s \geq 0
$$

or, equivalently,

$$
\lambda \geq \frac{b}{N \int_{0}^{1} \operatorname{sh}(s) d s} .
$$

Hence, the theorem is proved.

From Theorems 2.1 and 2.2, we have the following result.

Theorem 2.4. Assume $\left(\mathrm{H}_{1}\right)$ and $\left(\mathrm{H}_{2}\right)$. Then

(1) if $0<\lambda \leq b / M \int_{0}^{1} s h(s) d s$, then (2.1) has a positive radial solution;

(2) if $\lambda>b / M \int_{0}^{1} \operatorname{sh}(s) d s$, then (2.1) has at least two positive radial solutions, where $b$ and $h(t)$ are defined as in (2.8).

\section{Existence of a Double S-Shaped Bifurcation Curve}

\subsection{A Quadrature Method for (1.11)}

In this section, we analyze the positive solutions when $\Omega=(0,1), n=1$, and $f(s)=e^{\beta s /(\beta+s)}$. The structure of positive solutions for (1.11) is well known for the case $n=1$, as well as 


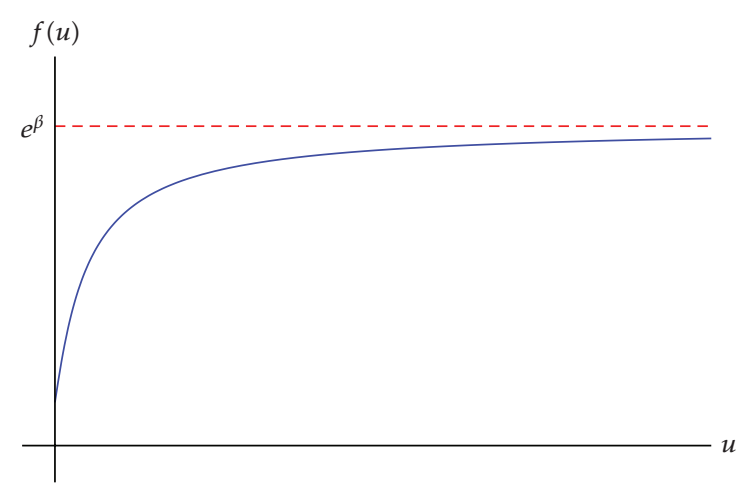

Figure 2: Graph of $f(u)$ when $\beta=2$.

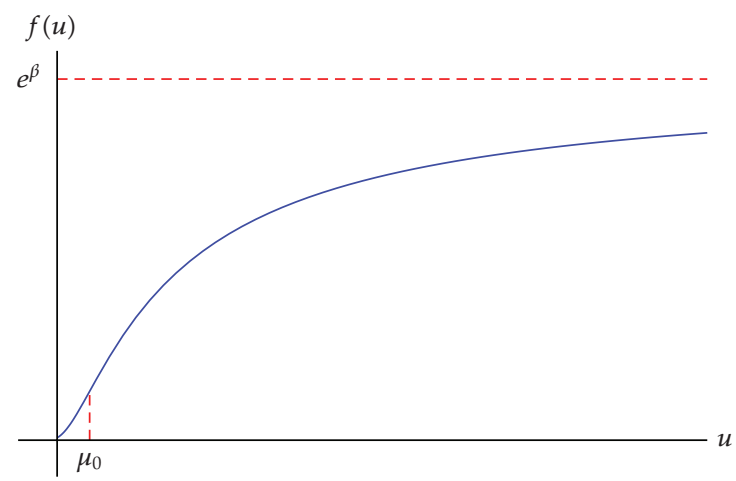

Figure 3: Graph of $f(u)$ when $\beta=5$.

higher dimensions. It has been studied by authors such as those of [16, 22], among others. For completeness, we include the Quadrature method developed by Laetsch in [23] to analyze positive solutions of the $n=1$ case, namely,

$$
\begin{gathered}
-u^{\prime \prime}=\lambda e^{\beta u /(\beta+u)}=: \lambda f(u), \quad x \in(0,1), \\
u(0)=0, \\
u(1)=0 .
\end{gathered}
$$

Define $F(u)=\int_{0}^{u} f(s) d s$, the primitive of $f(u)$. Figures 2 and 3 show $f(u)$ plotted for $\beta=2$ and $\beta=5$, respectively. Notice that $f(u)$ is concave on $[0, \infty)$ for $\beta \in(0,2]$. When $\beta \in(2, \infty)$, there exists a $\mu_{0} \in[0, \infty)$ such that $f(u)$ is convex on $\left[0, \mu_{0}\right)$ and concave on $\left(\mu_{0}, \infty\right)$. For all $\beta>0, f(u)$ is increasing on $[0, \infty]$ and bounded above by the horizonal asymptote, $y=e^{\beta}$. Also, $F(u)$ is shown in Figure 4 .

We present the main theorem of this subsection followed by computational results in the form of bifurcation diagrams.

Theorem 3.1 (see [16]). Let $\beta>0$, then (3.1)-(3.3) has a positive solution, $u(x)$, with $\|u\|_{\infty}=\rho$ if and only if $G(\rho):=\sqrt{2} \int_{0}^{\rho}(d s / \sqrt{F(\rho)-F(s)})=\sqrt{\lambda}$ for some $\lambda>0$. 


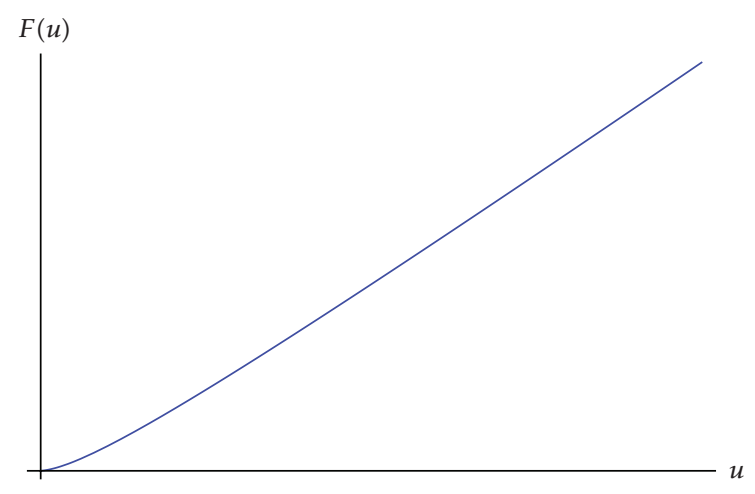

Figure 4: Graph of $F(u)$ when $\beta=5$.

Proof. Fix $\beta \in(0, \infty)$. $\left(\Rightarrow\right.$ :) Suppose that $u(x)$ is a positive solution to (3.1)-(3.3) with $\|u\|_{\infty}=\rho$. First note that (3.1) is an autonomous differential equation. Thus, if there exists a $x_{0} \in(0,1)$ such that $u^{\prime}\left(x_{0}\right)=0$ then both $v(x):=u\left(x_{0}+x\right)$ and $w(x):=u\left(x_{0}-x\right)$ satisfy the initial value problem,

$$
\begin{gathered}
-z^{\prime \prime}=\lambda f(z), \\
z(0)=u\left(x_{0}\right), \\
z^{\prime}(0)=0
\end{gathered}
$$

for all $x \in[0, d)$ where $d=\min \left\{x_{0}, 1-x_{0}\right\}$. By Picard's Existence and Uniqueness Theorem, $u\left(x_{0}+x\right) \equiv u\left(x_{0}-x\right)$. Hence, $u(x)$ must be symmetric about $x_{0}=1 / 2$ and $u^{\prime}(x) \geq 0 ; x \in\left[0, x_{0}\right]$ while $u^{\prime}(x) \leq 0 ; x \in\left[x_{0}, 1\right]$. Now, multiplying (3.1) by $u^{\prime}(x)$ yields,

$$
-\left[\frac{\left[u^{\prime}(x)\right]^{2}}{2}\right]^{\prime}=\lambda[F(u(x))]^{\prime} .
$$

Integrating throughout (3.5) from $x$ to $1 / 2$, we have,

$$
\frac{u^{\prime}(x)}{\sqrt{F(\rho)-F(u(x))}}=\sqrt{2 \lambda}, \quad x \in\left[0, \frac{1}{2}\right) .
$$

Integration of (3.6) from 0 to $x$ gives

$$
\int_{0}^{u(x)} \frac{d s}{\sqrt{F(\rho)-F(s)}}=\sqrt{2 \lambda} x, \quad x \in\left[0, \frac{1}{2}\right] .
$$

Using the fact that $u(1 / 2)=\rho,(3.7)$ becomes

$$
G(\rho):=\sqrt{2} \int_{0}^{\rho} \frac{d s}{\sqrt{F(\rho)-F(s)}}=\sqrt{\lambda} .
$$


$(\Leftarrow$ :) Suppose that there exists a $\lambda, \rho \in(0, \infty)$ such that $G(\rho)=\sqrt{\lambda}$. Now, define $u$ : $[0,1 / 2) \rightarrow \mathbb{R}$ by

$$
\int_{0}^{u(x)} \frac{d s}{\sqrt{F(\rho)-F(s)}}=\sqrt{2 \lambda} x .
$$

We will show that $u(x)$ is a positive solution of (3.1). It follows that the left-hand side of (3.9) is a differentiable function of $u$ which is strictly increasing from 0 to $1 / 2$ as $u$ increases from 0 to $\rho$. Hence, for each $x \in[0,1 / 2)$, there exists a unique $u(x)$ that satisfies

$$
\int_{0}^{u(x)} \frac{d s}{\sqrt{F(\rho)-F(s)}}=\sqrt{2 \lambda} x .
$$

By the Implicit Function Theorem, $u(x)$ is differentiable as a function of $x$. Differentiating (3.10), we have

$$
u^{\prime}(x)=\sqrt{2 \lambda[F(\rho)-F(u(x))]} ; \quad x \in\left[0, \frac{1}{2}\right] .
$$

Simplifying (3.11) gives

$$
-\frac{\left[u^{\prime}(x)\right]^{2}}{2}=\lambda[F(u(x))-F(\rho)] ; \quad x \in\left[0, \frac{1}{2}\right]
$$

Differentiating (3.12), we have

$$
-u^{\prime \prime}(x)=f(u(x))
$$

Thus, $u(x)$ satisfies the differential equation in (3.1). Also, it is clear that $u(0)=0$. Finally, defining $u(x)$ as a symmetric function on $(0,1)$, gives a positive solution to (3.1)-(3.3) with $\|u\|_{\infty}=\rho$ and $u(0)=0=u(1)$.

Remark 1 (see [16]). G( $\rho$ ) is well defined and the included improper integral is convergent since $f(\rho)>0$ and $F(u)$ is strictly increasing. Moreover, $G(\rho)$ is a continuous and differentiable function.

Also, analyzing (3.8) the following existence result was established in [16].

Theorem 3.2 (see [16]). For all $\beta>0,(1.11)$ has at least one positive solution for all $\lambda>0$.

\subsection{Computational Results for (1.11)}

In this subsection, we present the evolution of bifurcation curves for (1.11) suggested by our computational results. Mathematica was employed to plot $G(\rho)$ from Theorem 3.1 for various 


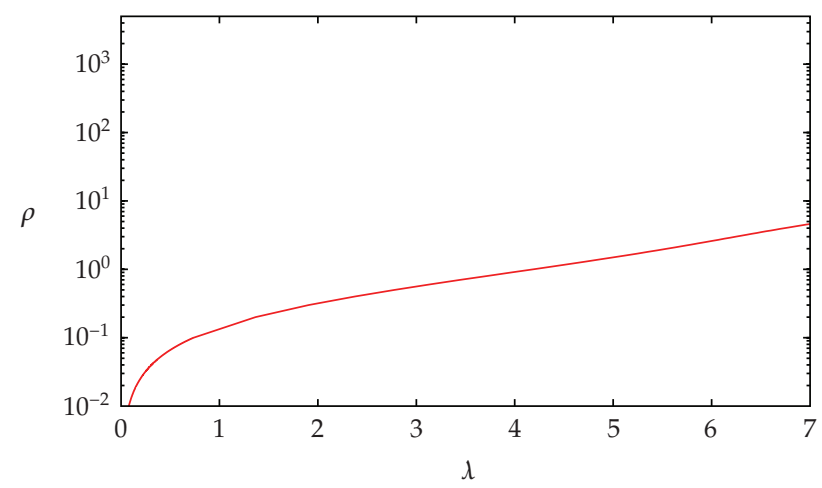

Figure 5: $\lambda$ versus $\rho$ for $\beta=3$.

values of $\beta$. Our results agree with those of previous authors such as [16], who was first to present them.

Case 1 (see [16]). If $\beta \in\left(0, \beta_{0}\right)$ (some $\beta_{0} \approx 4.25$ ) then (1.11) has a unique positive solution for all $\lambda>0$.

Figure 5 gives a typical bifurcation diagram for $\beta \in\left(0, \beta_{0}\right)$. Note that the following figures are log plots.

Case 2 (see [16]). If $\beta \in\left(\beta_{0}, \infty\right)$ then there exist $\lambda_{0}, \lambda_{1}>0$ such that if

(1) $\lambda_{0}<\lambda<\lambda_{1}$, then (1.11) has exactly 3 positive solutions;

(2) $\lambda=\lambda_{0}$ or $\lambda=\lambda_{1}$, then (1.11) has exactly 2 positive solutions;

(3) $0<\lambda<\lambda_{0}$ or $\lambda>\lambda_{1}$, then (1.11) has a unique positive solution.

Figure 6 gives a typical bifurcation diagram for $\beta \in\left(\beta_{0}, \infty\right)$.

\subsection{A Quadrature Method for (1.12)}

We will adapt the Quadrature method to analyze solutions of (1.12). Thus we study,

$$
\begin{gathered}
-u^{\prime \prime}=\lambda e^{\beta u /(\beta+u)}=: \lambda f(u), \quad x \in(0,1), \\
u(0)=0, \\
u^{\prime}(1)=-1,
\end{gathered}
$$

where $\lambda$ and $\beta$ are positive parameters. Again, define $F(u)=\int_{0}^{u} f(s) d s$, the primitive of $f(u)$. Using a similar argument to the one before, if there exists a $x_{0} \in(0,1)$ such that $u^{\prime}\left(x_{0}\right)=0$ then $u(x)$ is symmetric about $x_{0}$. Now, assume that $u(x)$ is a positive solution of (3.14)-(3.16) with $\rho:=\|u\|_{\infty}=u\left(x_{0}\right)$ for some $x_{0} \in(0,1)$ such that $u^{\prime}\left(x_{0}\right)=0$. Define $q:=u(1)$. Clearly, $u^{\prime}(x) \geq 0$ on $\left[0, x_{0}\right]$ and $u^{\prime}(x) \leq 0$ on $\left[x_{0}, 1\right]$. Hence, $u(x)$ must resemble Figure 7 .

Multiplying (3.14) by $u^{\prime}$, we have $-u^{\prime} u^{\prime \prime}=\lambda f(u) u^{\prime}$. Integrating with respect to $x$ gives

$$
\frac{-\left(u^{\prime}\right)^{2}}{2}=\lambda F(u)+K
$$




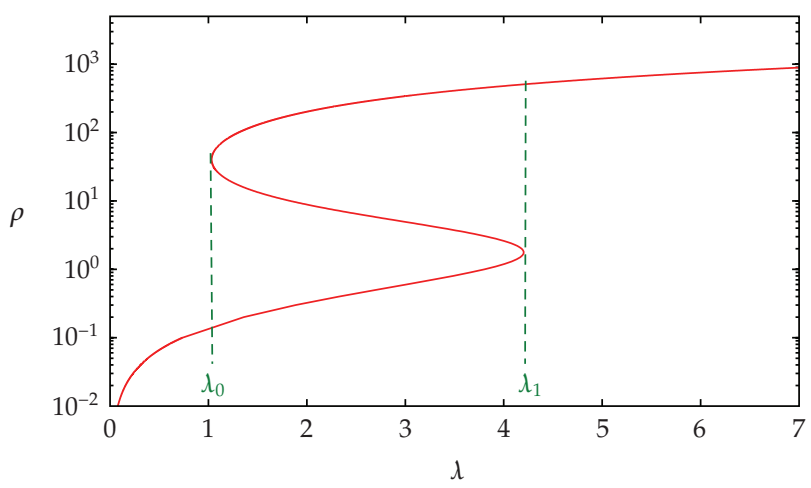

Figure 6: $\lambda$ versus $\rho$ for $\beta=7$.

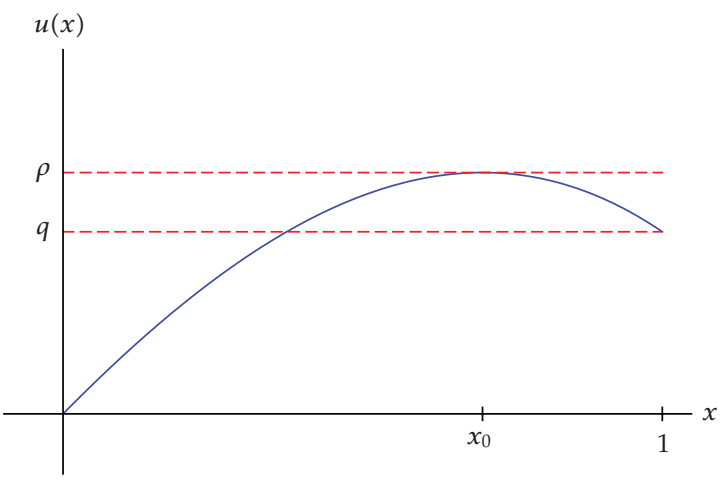

Figure 7: Typical solution of (3.14)-(3.16).

Substituting $x=x_{0}$ and $x=1$ into (3.17) while using $u^{\prime}\left(x_{0}\right)=0, u\left(x_{0}\right)=\rho, u(1)=q$, and $u^{\prime}(1)=-1$ yields

$$
\begin{gathered}
F(\rho)=-\frac{K}{\lambda}, \\
F(q)+\frac{1}{2 \lambda}=-\frac{K}{\lambda} .
\end{gathered}
$$

Combining (3.18) and (3.19) gives

$$
F(\rho)=F(q)+\frac{1}{2 \lambda}
$$

Substitution of (3.18) into (3.17) yields

$$
\frac{-\left(u^{\prime}\right)^{2}}{2}=\lambda(F(u)-F(\rho))
$$


Now, solving for $u^{\prime}$ in (3.21), we have

$$
\begin{array}{ll}
u^{\prime}(x)=\sqrt{2 \lambda[F(\rho)-F(u(x))]}, & x \in\left[0, x_{0}\right], \\
u^{\prime}(x)=-\sqrt{2 \lambda[F(\rho)-F(u(x))]}, & x \in\left[x_{0}, 1\right] .
\end{array}
$$

Integration of (3.22) combined with $u(0)=0$ and $u\left(x_{0}\right)=\rho$ gives,

$$
\begin{gathered}
\int_{0}^{u(x)} \frac{d s}{\sqrt{F(\rho)-F(s)}}=\sqrt{2 \lambda} x, \quad x \in\left[0, x_{0}\right], \\
\int_{\rho}^{u(x)} \frac{d s}{\sqrt{F(\rho)-F(s)}}=-\sqrt{2 \lambda}\left(x-x_{0}\right), \quad x \in\left[x_{0}, 1\right] .
\end{gathered}
$$

We substitute $x=x_{0}$ into (3.23) and $x=1$ into (3.24) giving

$$
\begin{gathered}
\int_{0}^{\rho} \frac{d s}{\sqrt{F(\rho)-F(s)}}=\sqrt{2 \lambda} x_{0}, \\
\int_{\rho}^{q} \frac{d s}{\sqrt{F(\rho)-F(s)}}=-\sqrt{2 \lambda}\left(1-x_{0}\right) .
\end{gathered}
$$

Subtract (3.26) from (3.25) yields,

$$
2 \int_{0}^{\rho} \frac{d s}{\sqrt{F(\rho)-F(s)}}-\int_{0}^{q} \frac{d s}{\sqrt{F(\rho)-F(s)}}=\sqrt{2 \lambda}
$$

Solving (3.21) for $\sqrt{2 \lambda}$ and using $u^{\prime}(1)=-1$ and $u(1)=q$, we have

$$
\sqrt{2 \lambda}=\frac{1}{\sqrt{F(\rho)-F(q)}}
$$

Combining (3.28) with (3.27) we define,

$$
\widetilde{H}(\rho, q):=2 \int_{0}^{\rho} \frac{d s}{\sqrt{F(\rho)-F(s)}}-\int_{0}^{q} \frac{d s}{\sqrt{F(\rho)-F(s)}}-\frac{1}{\sqrt{F(\rho)-F(q)}} .
$$


Now, for each $\rho \in(0, \infty)$, we need to find a $q=q(\rho) \in[0, \rho)$ such that $\widetilde{H}(\rho, q(\rho))=0$. If for fixed $\rho \in(0, \infty)$ there is a unique $q(\rho) \in[0, \rho)$ with $\widetilde{H}(\rho, q(\rho))=0$ then

$$
2 \int_{0}^{\rho} \frac{d s}{\sqrt{F(\rho)-F(s)}}-\int_{0}^{q(\rho)} \frac{d s}{\sqrt{F(\rho)-F(s)}}=\frac{1}{\sqrt{F(\rho)-F(q(\rho))}}=\sqrt{2 \lambda}
$$

will be satisfied for a unique $\lambda \in(0, \infty)$. As a result, we need to analyze the existence and uniqueness of such a $q=q(\rho)$. The following lemma lists several properties of $\widetilde{H}(\rho, q)$.

Lemma B. (1) For every $\rho>0, \widetilde{H}(\rho, q) \rightarrow-\infty$ as $q \rightarrow \rho$.

(2) For all $\rho>0$ and $q \in[0, \rho)$ one has that $\widetilde{H}_{q}(\rho, q)<0$.

(3) $\widetilde{H}(\rho, 0) \rightarrow \infty$ as $\rho \rightarrow \infty$.

(4) $\widetilde{H}(\rho, 0) \rightarrow-\infty$ as $\rho \rightarrow 0$.

Proof. (1) It follows from the fact that $F(u)$ is increasing and the Mean Value Theorem.

(2) Fix $\rho>0$. Then for all $q \in[0, \rho)$,

$$
\widetilde{H}_{q}(\rho, q)=-\frac{1}{\sqrt{F(\rho)-F(q)}}-\frac{f(q)}{2[F(\rho)-F(q)]^{3 / 2}}<0
$$

(3) For all $\rho>0$,

$$
\widetilde{H}(\rho, 0)=2 \int_{0}^{\rho} \frac{d s}{\sqrt{F(\rho)-F(s)}}-\frac{1}{\sqrt{F(\rho)}}
$$

$\Rightarrow \widetilde{H}(\rho, 0) \rightarrow \infty$ as $\rho \rightarrow \infty$.

(4) Again, this follows from the Mean Value Theorem and monotonicity of $F(u)$.

Notice that if $\widetilde{H}(\rho, 0)>0$, then there will be a unique $q(\rho) \in[0, \rho)$ such that $\widetilde{H}(\rho, q(\rho))=0$. From Lemma B, $\widetilde{H}(\rho, q)$ must resemble Figure 8 .

Figures 9 and 10 show what $\widetilde{H}(\rho, 0)$ resembles $\beta \in(0,4]$ and $\beta \in(4, \infty)$, respectively.

For $\beta \in(0,4]$ there exists a unique $\rho_{0}>0$ such that if $\rho \geq \rho_{0}$ then $\widetilde{H}(\rho, 0) \geq 0$ and if $\rho<\rho_{0}$ then $\widetilde{H}(\rho, 0)<0$. In the second case, $\beta \in(4, \infty)$, the shape of $\widetilde{H}(\rho, 0)$ changes from that of the first case with the addition of both a local maximum and a local minimum. However, based on our computations, we conjecture that there exists a unique $\rho_{0}>0$ such that if $\rho \geq \rho_{0}$ then $\widetilde{H}(\rho, 0) \geq 0$ and if $\rho<\rho_{0}$ then $\widetilde{H}(\rho, 0)<0$. Hence, for each $\rho \in\left[\rho_{0}, \infty\right)$ there is a unique $q(\rho) \in[0, \rho)$ such that $\widetilde{H}(\rho, q(\rho))=0$. Next we define

$$
H(\rho, q(\rho)):=\frac{1}{2[F(\rho)-F(q(\rho))]}
$$

for all $\rho \in\left[\rho_{0}(\beta), \infty\right)$ and $q(\rho) \in[0, \rho)$ and present the main theorem of the section. 
Boundary Value Problems

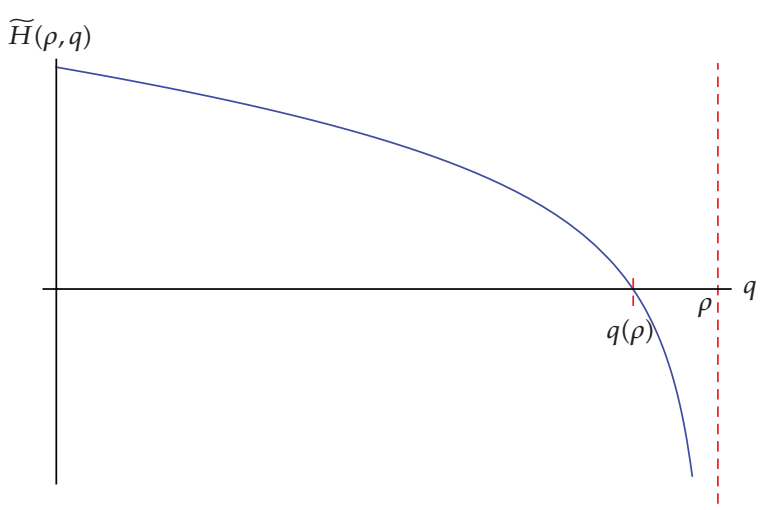

Figure 8: Graph of $\widetilde{H}(\rho, q)$.

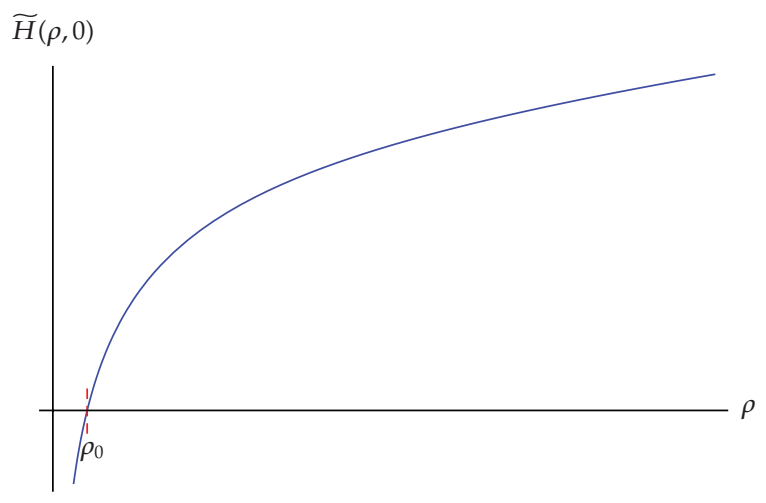

Figure 9: Graph of $\widetilde{H}(\rho, 0)$ for $\beta \in(0,4]$.

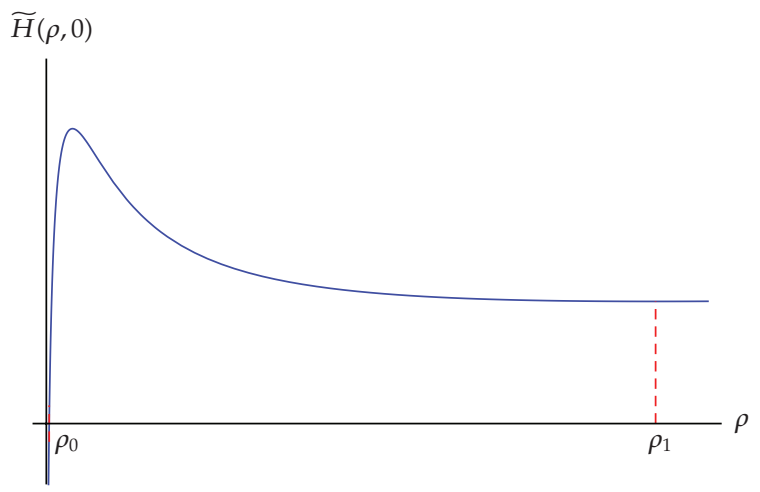

Figure 10: $\widetilde{H}(\rho, 0)$ for $\beta \in(4, \infty)$.

Theorem 3.3. Let $\beta>0$, then (3.14)-(3.16) has a positive solution, $u(x)$, with $\|u\|_{\infty}=\rho \in S(\beta):=$ $\left[\rho_{0}(\beta), \infty\right) \Leftrightarrow H(\rho, q(\rho))=\lambda$ for some $\lambda>0$ where $q=q(\rho) \in[0, \rho)$ is the unique solution of $\widetilde{H}(\rho, q(\rho))=0$.

Proof. Fix $\beta \in(0, \infty)$. $(\Rightarrow$ :) is completed through preceding discussion. 
$(\Leftarrow:)$ Suppose that there exist $\lambda \in(0, \infty), \rho \in S(\beta)$ such that $H(\rho, q(\rho))=\lambda$ where $q(\rho) \in[0, \rho)$ is the unique solution of $\widetilde{H}(\rho, q(\rho))=0$. Define $u(x):(0,1) \rightarrow \mathbb{R}$ by

$$
\begin{gathered}
\int_{0}^{u(x)} \frac{d s}{\sqrt{F(\rho)-F(s)}}=\sqrt{2 \lambda x}, \quad x \in\left[0, x_{0}\right], \\
\int_{0}^{u(x)} \frac{d s}{\sqrt{F(\rho)-F(s)}}=-\sqrt{2 \lambda}\left(x-x_{0}\right), \quad x \in\left[x_{0}, 1\right] .
\end{gathered}
$$

We will show that $u(x)$ is a positive solution to (3.14)-(3.16). Notice that the turning point of $u(x), x_{0}$, is given by

$$
x_{0}=\frac{1}{\sqrt{2 \lambda}} \int_{0}^{\rho} \frac{d s}{\sqrt{F(\rho)-F(s)}} .
$$

Clearly, for fixed $\lambda$,

$$
\frac{1}{\sqrt{2 \lambda}} \int_{0}^{u(x)} \frac{d s}{\sqrt{F(\rho)-F(s)}}
$$

is a differentiable function of $u$ and is strictly increasing from 0 to $x_{0}$ as $u$ increases from 0 to $\rho$. Hence, for each $x \in\left[0, x_{0}\right]$ there exists a unique $u(x)$ such that

$$
\int_{0}^{u(x)} \frac{d s}{\sqrt{F(\rho)-F(s)}}=\sqrt{2 \lambda} x .
$$

By the Implicit Function Theorem, $u(x)$ is differentiable with respect to $x$. This implies that,

$$
u^{\prime}(x)=\sqrt{2 \lambda[F(\rho)-F(u(x))]}, \quad x \in\left[0, x_{0}\right] .
$$

A similar argument can be made to show that

$$
u^{\prime}(x)=-\sqrt{2 \lambda[F(\rho)-F(u(x))]}, \quad x \in\left[x_{0}, 1\right] .
$$

From (3.38) and (3.39), we have,

$$
\frac{\left[u^{\prime}(x)\right]^{2}}{2}=\lambda[F(\rho)-F(u(x))], \quad x \in[0,1] .
$$


Differentiating (3.40) gives

$$
\begin{array}{ll}
-u^{\prime \prime} u^{\prime}=\lambda f(u) u^{\prime}, & x \in(0,1), \\
\Longrightarrow-u^{\prime \prime}=\lambda f(u), & x \in(0,1) .
\end{array}
$$

Hence, $u(x)$ satisfies (3.14). It only remains to show that $u(x)$ satisfies (3.15) and (3.16). But, it is clear that $u(0)=0$. Also, since $H(\rho, q(\rho))=\lambda$, we have

$$
2 \lambda=\frac{1}{F(\rho)-F(q(\rho))}
$$

or equivalently,

$$
F(\rho)-F(q(\rho))=\frac{1}{2 \lambda}
$$

Substituting $x=1$ into (3.39) gives

$$
u^{\prime}(1)=-\sqrt{2 \lambda} \sqrt{F(\rho)-F(q(\rho))} .
$$

Combining (3.43) and (3.44),

$$
u^{\prime}(1)=-1
$$

Hence, $u(x)$ satisfies both (3.15) and (3.16).

To conclude the subsection, we present several results that detail the global behavior of $H(\rho, q(\rho))$.

Remark 1. Note that given $\beta>0$, (3.14)-(3.16) has no positive solution with $\|u\|_{\infty}<\rho_{0}(\beta)$.

Remark 2. For every $\beta>0, H(\rho, q(\rho)) \leq[G(\rho)]^{2}$ for all $\rho>\rho_{0}(\beta)$. Moreover, equality is achieved if and only if $\rho=\rho_{0}(\beta)$ in which case, $q(\rho)=0$.

This follows from observing that

$$
\begin{aligned}
H(\rho, q(\rho)) & =\frac{1}{2[F(\rho)-F(q(\rho))]} \\
& =\left[\sqrt{2} \int_{0}^{\rho} \frac{d s}{\sqrt{F(\rho)-F(s)}}-\frac{1}{\sqrt{2}} \int_{0}^{q(\rho)} \frac{d s}{\sqrt{F(\rho)-F(s)}}\right]^{2} \\
& \leq\left[\sqrt{2} \int_{0}^{\rho} \frac{d s}{\sqrt{F(\rho)-F(s)}}\right]^{2}=[G(\rho)]^{2} .
\end{aligned}
$$


Theorem 3.4. Let $\beta>0$. If $\rho \geq \rho_{0}(\beta), q(\rho)$, and $\lambda$ are as in the previous theorem with $H(\rho, q(\rho))=$ $\lambda$ then
(a) $q(\rho) \rightarrow \rho$ as $\rho \rightarrow \infty$, hence, $x_{0} \rightarrow 1$ as $\rho \rightarrow \infty$;
(b) $\lambda \rightarrow \infty$ as $\rho \rightarrow \infty$;
(c) $\lambda \geq 2 \rho / e^{2 \beta}$.

Proof. Fix $\beta>0$ and let $\rho \geq \rho_{0}(\beta), q(\rho)$, and $\lambda$ be as in the previous theorem with $H(\rho, q(\rho))=$ $\lambda$.

(a) Claim: $\rho-q(\rho) \leq e^{\beta} / 4 \rho$

With this claim, it is clear that for fixed $\beta,[\rho-q(\rho)] \rightarrow 0$ as $\rho \rightarrow \infty$. Now to prove the claim. Since $H(\rho, q(\rho))=\lambda$, we have that

$$
\int_{0}^{\rho} \frac{d s}{\sqrt{F(\rho)-F(s)}}+\int_{q(\rho)}^{\rho} \frac{d t}{\sqrt{F(\rho)-F(t)}}=\frac{1}{\sqrt{F(\rho)-F(q(\rho))}} .
$$

By the Mean Value Theorem, there exist $\theta_{1} \in(s, \rho), \theta_{2} \in(t, \rho)$, and $\theta_{3} \in(q(\rho), \rho)$ with $s \in$ $(0, \rho)$ and $t \in(q(\rho), \rho)$ such that

$$
\int_{0}^{\rho} \frac{d s}{\sqrt{f\left(\theta_{1}\right)} \sqrt{\rho-s}}+\int_{q(\rho)}^{\rho} \frac{d t}{\sqrt{f\left(\theta_{2}\right)} \sqrt{\rho-t}}=\frac{1}{\sqrt{f\left(\theta_{3}\right)} \sqrt{\rho-q(\rho)}}
$$

Now, since $f(u)$ is monotone increasing, we have that $f\left(\theta_{1}\right), f\left(\theta_{2}\right) \leq f(\rho)$ and

$$
\frac{1}{\sqrt{f(\rho)}} \int_{0}^{\rho} \frac{d s}{\sqrt{\rho-s}}+\frac{1}{\sqrt{f(\rho)}} \int_{q(\rho)}^{\rho} \frac{d t}{\sqrt{\rho-t}} \leq \frac{1}{\sqrt{f\left(\theta_{3}\right)} \sqrt{\rho-q(\rho)}}
$$

A change of variables in the integrals of (3.49) yields,

$$
\frac{\sqrt{\rho}}{\sqrt{f(\rho)}} \int_{0}^{1} \frac{d v}{\sqrt{1-v}}+\frac{\sqrt{\rho}}{\sqrt{f(\rho)}} \int_{q / \rho}^{1} \frac{d w}{\sqrt{1-w}} \leq \frac{1}{\sqrt{f\left(\theta_{3}\right)} \sqrt{\rho-q(\rho)}}
$$

This implies that,

$$
\rho-q(\rho) \leq \frac{f(\rho)}{4 f\left(\theta_{3}\right) \rho} \leq \frac{e^{\beta}}{4 \rho}
$$

since $f(\rho) \leq e^{\beta}$ and $f\left(\theta_{3}\right) \geq 1$. 


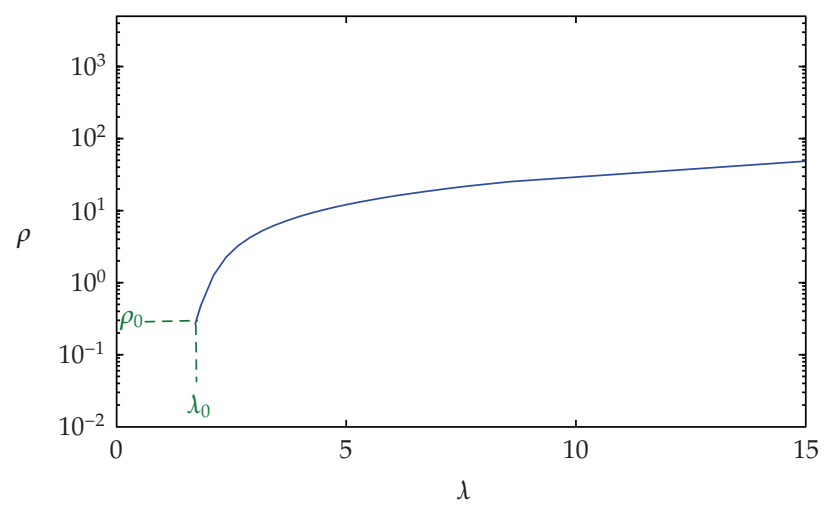

Figure 11: Graph of $\lambda$ versus $\rho$ for $\beta=2$.

(b) By the Mean Value Theorem there exists a $\theta \in(q(\rho), \rho)$ such that

$$
\lambda=\frac{1}{2 f(\theta)[\rho-q(\rho)]} .
$$

But, $1 \leq f(\theta) \leq e^{\beta}$, which implies that

$$
\frac{1}{2 e^{\beta}[\rho-q(\rho)]} \leq \lambda \leq \frac{1}{2[\rho-q(\rho)]}
$$

Part (a) combined with (3.53) completes (b).

(c) Finally (3.51) combined with (3.53) yields (c).

Corollary 3.5. For every $\beta>0$, there exists a $\lambda_{1}>0$ such that (3.14)-(3.16) has no positive solution for all $\lambda<\lambda_{1}$.

\subsection{Computational Results for (1.12) and (1.10)}

This subsection will present computational results first for (1.12) then for our original problem, (1.10). In order to produce bifurcation diagrams, Mathematica was employed in a two-step process. Recalling Theorem 3.3 from Section 3.3, for fixed $\beta>0$ the corresponding unique $\rho_{0}(\beta)$ is first found using a standard root-finding algorithm. Then for each $\rho \geq \rho_{0}(\beta)$, the same root-finding algorithm is employed to solve $\widetilde{H}(\rho, q(\rho))=0$ for the unique $q$ value. Finally, $H(\rho, q(\rho))$ is evaluated for the given $\rho$ and its unique $q(\rho)$ to obtain the corresponding unique $\lambda$. The result is a bifurcation diagram portraying $\lambda$ versus $\rho$. Due to the improper integrals in $H(\rho, q(\rho))$, this procedure is computationally expensive. The numerical investigations suggest the following evolution of bifurcation diagrams.

Case 1. For $\beta \in\left(0, \beta_{1}\right)$ (for some $\left.\beta_{1}<4\right)$, there exists a $\lambda_{0}>0$ such that if

(1) $\lambda \geq \lambda_{0}$, then (1.12) has a unique solution;

(2) $\lambda<\lambda_{0}$, then (1.12) has no positive solution.

Figure 11 illustrates Case 1. 


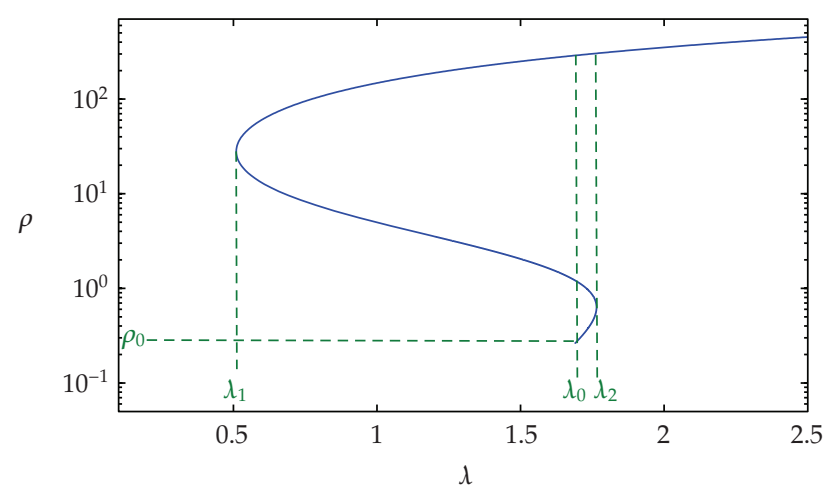

Figure 12: Graph of $\lambda$ versus $\rho$ for $\beta=6$.

Case 2. For $\beta \in\left[\beta_{1}, \infty\right)$, there exists $\lambda_{0}, \lambda_{1}, \lambda_{2}>0$ such that if

(1) $\lambda_{0} \leq \lambda<\lambda_{2}$, then (1.12) has exactly 3 positive solutions;

(2) $\lambda_{1}<\lambda<\lambda_{0}$ or $\lambda=\lambda_{2}$, then (1.12) has exactly 2 positive solutions;

(3) $\lambda>\lambda_{2}$ or $\lambda=\lambda_{1}$, then (1.12) has a unique positive solution.

Figure 12 shows a typical bifurcation diagram for Case 2.

Notice that $\left(\lambda_{0}, \rho_{0}\right)$ corresponds to the case when $q(\rho)=u(1)=0$ and thus satisfies both (1.11) and (1.12). We would then expect this to be the point at which the branch of solutions of (1.10) bifurcates into the separate cases. To conclude the section, we present the computational results for (1.10) by combining the solutions of (1.11) in Section 3.1 and (1.12) in Section 3.3.

Theorem 3.6. For $\beta>0$, (1.10) has at least one positive solution for every $\lambda>0$.

Case 1. For $\beta \in\left(0, \beta_{1}\right)$, there exists a $\lambda_{0}>0$ such that if

(1) $\lambda>\lambda_{0}$, then (1.10) has 2 positive solutions,

(2) $\lambda \leq \lambda_{0}$, then (1.10) has a unique positive solution.

Figure 13 shows the complete bifurcation diagram of (1.10) for $\beta=2$.

Case 2. For $\beta \in\left[\beta_{1}, \beta_{0}\right)$, there exists $\lambda_{0}, \lambda_{1}, \lambda_{2}>0$ such that if

(1) $\lambda_{0}<\lambda<\lambda_{2}$, then (1.10) has exactly 4 positive solutions;

(2) $\lambda_{1}<\lambda \leq \lambda_{0}$ or $\lambda=\lambda_{0}, \lambda_{2}$, then (1.10) has exactly 3 positive solutions;

(3) $\lambda>\lambda_{2}$ or $\lambda=\lambda_{1}$, then (1.10) has exactly 2 positive solutions;

(4) $\lambda<\lambda_{1}$ then (1.10) has a unique positive solution.

Case 2 is illustrated in Figure 14.

Case 3. For $\beta \in\left[\beta_{0}, \beta_{2}\right)$ (some $\beta_{2} \in(6,6.5)$ ), there exists $\lambda_{i}>0$ for $i=0,1,2,3,4$ such that if

(1) $\lambda_{0}<\lambda<\lambda_{2}$ or $\lambda_{3}<\lambda<\lambda_{4}$, then (1.10) has exactly 4 positive solutions;

(2) $\lambda_{1}<\lambda \leq \lambda_{0}$ or $\lambda=\lambda_{2}, \lambda_{3}, \lambda_{4}$, then (1.10) has exactly 3 positive solutions; 


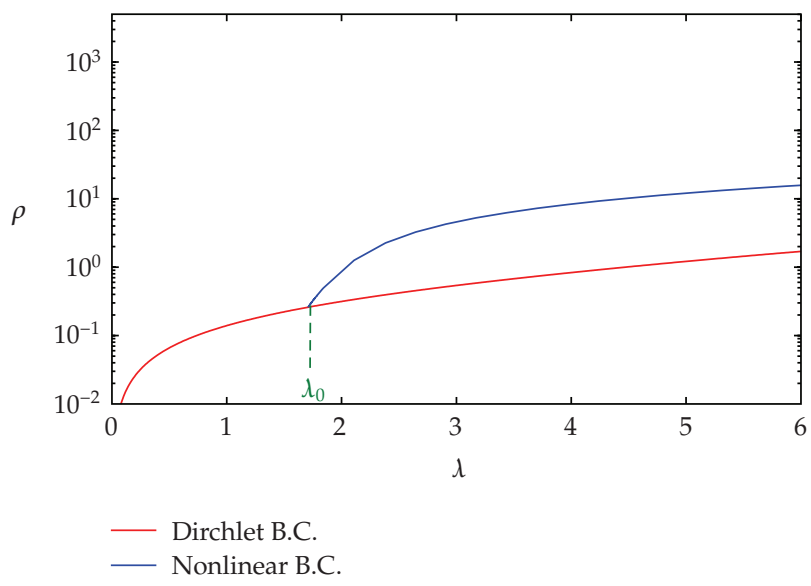

Figure 13: Graph of $\lambda$ versus $\rho$ for $\beta=2$.

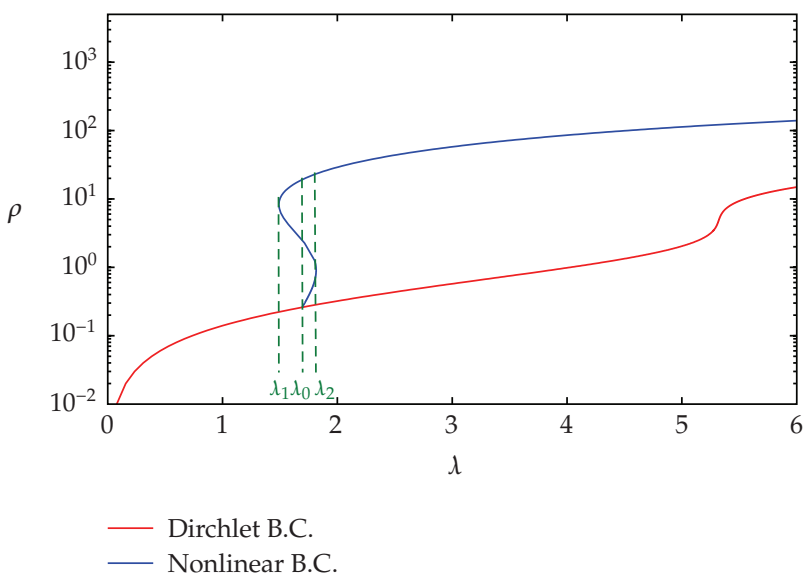

Figure 14: Graph of $\lambda$ versus $\rho$ for $\beta=4$.

(3) $\lambda_{2}<\lambda<\lambda_{3}$ or $\lambda>\lambda_{4}$ or $\lambda=\lambda_{1}$, then (1.10) has exactly 2 positive solutions;

(4) $\lambda<\lambda_{1}$, then (1.10) has a unique positive solution.

Case 3 is shown in Figure 15. Notice that the bifurcation diagram contains two Sshaped curves that do not overlap.

Case 4. For $\beta \in\left[\beta_{2}, \infty\right)$, there exists $\lambda_{i}>0$ for $i=0,1,2,3,4$ such that if

(1) $\lambda_{0}<\lambda<\lambda_{3}$, then (1.10) has exactly 6 positive solutions;

(2) $\lambda_{2}<\lambda \leq \lambda_{0}$ or $\lambda=\lambda_{3}$, then (1.10) has exactly 5 positive solutions;

(3) $\lambda_{3}<\lambda<\lambda_{4}$ or $\lambda=\lambda_{2}$, then (1.10) has exactly 4 positive solutions;

(4) $\lambda_{1}<\lambda<\lambda_{2}$ or $\lambda=\lambda_{4}$, then (1.10) has exactly 3 positive solutions;

(5) $\lambda>\lambda_{4}$ or $\lambda=\lambda_{1}$, then (1.10) has exactly 2 positive solutions;

(6) $\lambda<\lambda_{1}$ then (1.10) has a unique positive solution. 


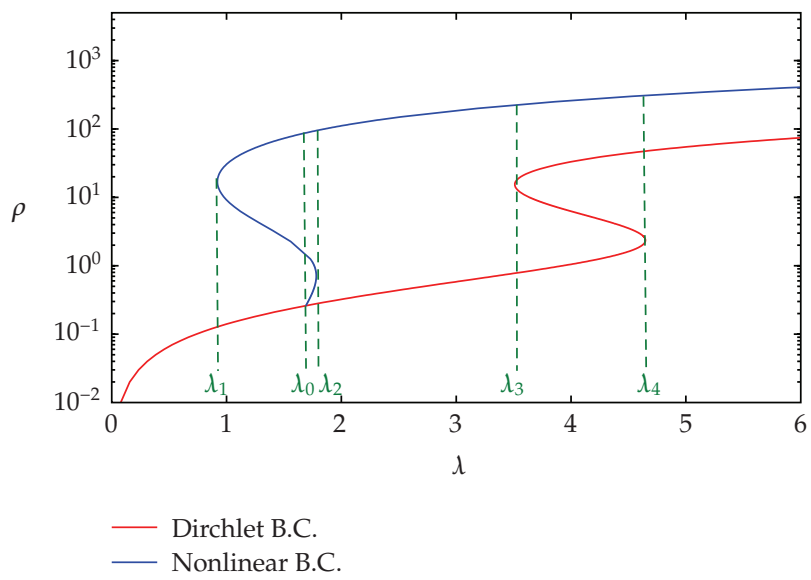

Figure 15: Graph of $\lambda$ versus $\rho$ for $\beta=5$.

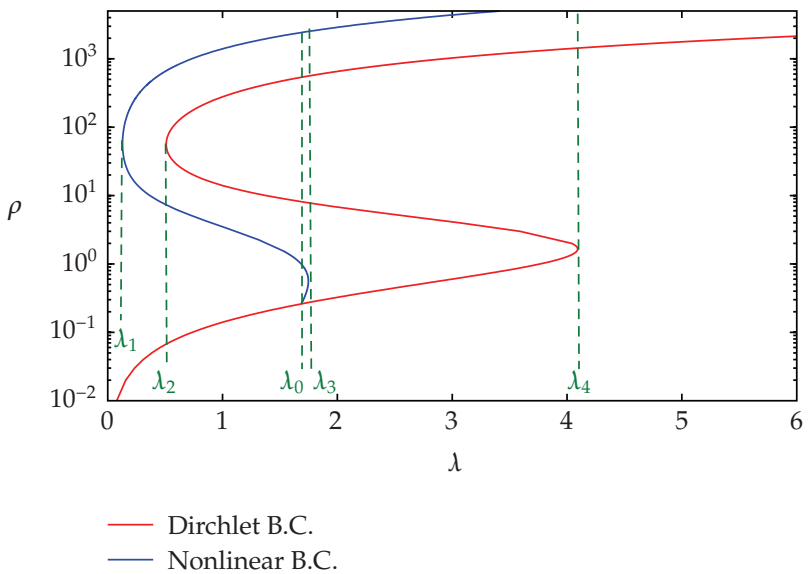

Figure 16: Graph of $\lambda$ versus $\rho$ for $\beta=8$.

Figure 16 exemplifies Case 4 with the complete bifurcation diagram for $\beta=8$. Again the double $S$-shape appears but in this case the Ss overlap, yielding exactly 6 positive solutions for a certain range of $\lambda$.

\section{Acknowledgment}

Eun Kyoung Lee was supported by the National Research Foundation of Korea Grant funded by the Korean Government [NRF-2009-353-C00042].

\section{References}

[1] R. S. Cantrell and C. Cosner, Spatial Ecology via Reaction-Diffusion Equations, Wiley Series in Mathematical and Computational Biology, John Wiley \& Sons, Chichester, UK, 2003.

[2] R. S. Cantrell and C. Cosner, "Density dependent behavior at habitat boundaries and the Allee effect," Bulletin of Mathematical Biology, vol. 69, no. 7, pp. 2339-2360, 2007. 
[3] R. S. Cantrell, C. Cosner, and S. Martínez, "Global bifurcation of solutions to diffusive logistic equations on bounded domains subject to nonlinear boundary conditions," Proceedings of the Royal Society of Edinburgh. Section A, vol. 139, no. 1, pp. 45-56, 2009.

[4] J. Goddard II and R. Shivaji, "A population model with nonlinear boundary conditions and constant yield harvesting," submitted.

[5] J. Bebernes and D. Eberly, Mathematical Problems from Combustion Theory, vol. 83 of Applied Mathematical Sciences, Springer, New York, NY, USA, 1989.

[6] Y. Du and Y. Lou, "Proof of a conjecture for the perturbed Gelfand equation from combustion theory," Journal of Differential Equations, vol. 173, no. 2, pp. 213-230, 2001.

[7] S. P. Hastings and J. B. McLeod, "The number of solutions to an equation from catalysis," Proceedings of the Royal Society of Edinburgh. Section A, vol. 101, no. 1-2, pp. 15-30, 1985.

[8] P. Korman and Y. Li, "On the exactness of an S-shaped bifurcation curve," Proceedings of the American Mathematical Society, vol. 127, no. 4, pp. 1011-1020, 1999.

[9] P. Korman and J. Shi, "Instability and exact multiplicity of solutions of semilinear equations," in Proceedings of the Conference on Nonlinear Differential Equations (Coral Gables, FL, 1999), vol. 5 of Electronic Journal of Differential Equations Conference, pp. 311-322, Southwest Texas State University, San Marcos, Tex, USA.

[10] J. R. Parks, "Criticality criteria for various configurations of a self-heating chemical as functions of activation energy and temperature of assembly," The Journal of Chemical Physics, vol. 34, no. 1, pp. 46-50, 1961.

[11] S. V. Parter, "Solutions of a differential equation arising in chemical reactor processes," SIAM Journal on Applied Mathematics, vol. 26, pp. 687-716, 1974.

[12] D. H. Sattinger, "A nonlinear parabolic system in the theory of combustion," Quarterly of Applied Mathematics, vol. 33, pp. 47-61, 1975/76.

[13] K. K. Tam, "Construction of upper and lower solutions for a problem in combustion theory," Journal of Mathematical Analysis and Applications, vol. 69, no. 1, pp. 131-145, 1979.

[14] S. H. Wang, "On S-shaped bifurcation curves," Nonlinear Analysis: Theory, Methods \& Applications, vol. 22, no. 12, pp. 1475-1485, 1994.

[15] S.-H. Wang and T.-S. Yeh, "Exact multiplicity of solutions and S-shaped bifurcation curves for the p-Laplacian perturbed Gelfand problem in one space variable," Journal of Mathematical Analysis and Applications, vol. 342, no. 2, pp. 1175-1191, 2008.

[16] K. J. Brown, M. M. A. Ibrahim, and R. Shivaji, "S-shaped bifurcation curves," Nonlinear Analysis: Theory, Methods \& Applications, vol. 5, no. 5, pp. 475-486, 1981.

[17] R. Shivaji, "A remark on the existence of three solutions via sub-super solutions," in Nonlinear Analysis and Applications (Arlington, Tex., 1986), vol. 109 of Lecture Notes in Pure and Applied Mathematics, pp. 561-566, Dekker, New York, NY, USA, 1987.

[18] H. Wang, "On the existence of positive solutions for semilinear elliptic equations in the annulus," Journal of Differential Equations, vol. 109, no. 1, pp. 1-7, 1994.

[19] Y.-H. Lee, "Multiplicity of positive radial solutions for multiparameter semilinear elliptic systems on an annulus," Journal of Differential Equations, vol. 174, no. 2, pp. 420-441, 2001.

[20] H. Amann, "Fixed point equations and nonlinear eigenvalue problems in ordered Banach spaces," SIAM Review, vol. 18, no. 4, pp. 620-709, 1976.

[21] D. J. Guo and V. Lakshmikantham, Nonlinear Problems in Abstract Cones, Academic Press, Orlando, Fla, USA, 1998

[22] A. Castro and R. Shivaji, "Nonnegative solutions for a class of nonpositone problems," Proceedings of the Royal Society of Edinburgh. Section A, vol. 108, no. 3-4, pp. 291-302, 1988.

[23] T. Laetsch, "The number of solutions of a nonlinear two point boundary value problem," Indiana University Mathematics Journal, vol. 20, pp. 1-13, 1970. 